\title{
Control Strategy and Communication Architecture for Power Sharing in Microgrids
}

\author{
Ricardo E. Pérez-Guzmán, Yamisleydi Salgueiro, Marco Rivera \\ Facultad de Ingeniería \\ Universidad de Talca \\ Curicó, Chile \\ riperez@utalca.cl,ysalgueiro@utalca.cl,marcoriv@utalca.cl
}

\author{
Patrick W. Wheeler \\ Dept. of Electrical and Electronic Engineering. \\ University of Nottingham \\ Nottingham NG7 2RD U.K. \\ pat.wheeler@nottingham.ac.uk
}

\begin{abstract}
The increasing needs of control, security and generation capacity of microgrids, has raised the complexity of the network and have allowed the evolution of architecture as a cluster. This increase in complexity demands control strategies in real-time, to ensure the proper functioning of the electrical system. In this research, a new communication strategy based on OFDM technology is proposed for power sharing in a microgrid cluster. To this, two modulation technologies are used based on OFDM (QPSK and 64-QAM) and the results are compared. The communication channel has been designed according to the properties of dispersion, attenuation, and noise, which are found in the applied environment. The study is based on a simulation model implemented in MATLAB, where was possible to verify the correct performance of the converters when the communications system sends the reference, achieving an error below $1 \%$.
\end{abstract}

Keywords-Communicatios; 64 QAM; microgrid cluster, OFDM; power sharing; power converters; QPSK.

\section{INTRODUCTION}

Electric power systems need substantial changes, due to the growing demands of a more reliable and secure network. One of the most important tasks for the utilities is to increase the reliability of the electrical system. Natural disasters, cyber attacks, or human errors, have caused widespread power outages and significant economic losses [1].

To address this problem, an alternative that adds intelligence and flexibility to the system are microgrids. They consist in a smaller and localized version of the smart grid, which brings distributed energy resources (DG) [2] to consumers, improving overall efficiency. The microgrid (MG) is able to operate connected, or disconnected to the main network, working in island mode. The cluster architecture used in this investigation is presented in Fig. 1. It shows the interconnection of several sources of generation, storage and loads, sharing power to the AC bus. Each DG has a power converter and a local controller (LC), which maintain some constant output parameters in the converter.

The DG sources increase the dimensions of the electrical system and the development of more complex devices, limits the efficient use of a microgrid [1], [3]. The interconnection of several microgrids to form a cluster is considered a possible solution, to improve the reliability, stability and security of the energy supply [4] and makes the MG a much more scalable element. The interconnection of multiple DGs requires efficient real-time control strategies to share energy reliably and securely. These control strategies are usually not designed according to these elements which may incur on important problems to be faced by control strategies.

Assigning the control task to different units which depend on the operation of the microgrid, compose the hierarchy of distributed control (primary, secondary and tertiary control). In this way, a cluster of microgrids can collaborate with each other to deal with contingencies and provide enough independence of each layer, exchanging power in the MG. Currently, two fundamental techniques to control the exchange of energy in a cluster of microgrids stand out. The first is called "droop control" and is characterized by sharing a linear relationship between voltage and current, or between power and voltage. This relationship allows the shared use of energy, simulating the steady state characteristics of a synchronous generator [4]. It offers a plug and play function and does not require communication, making it a more economical alternative.

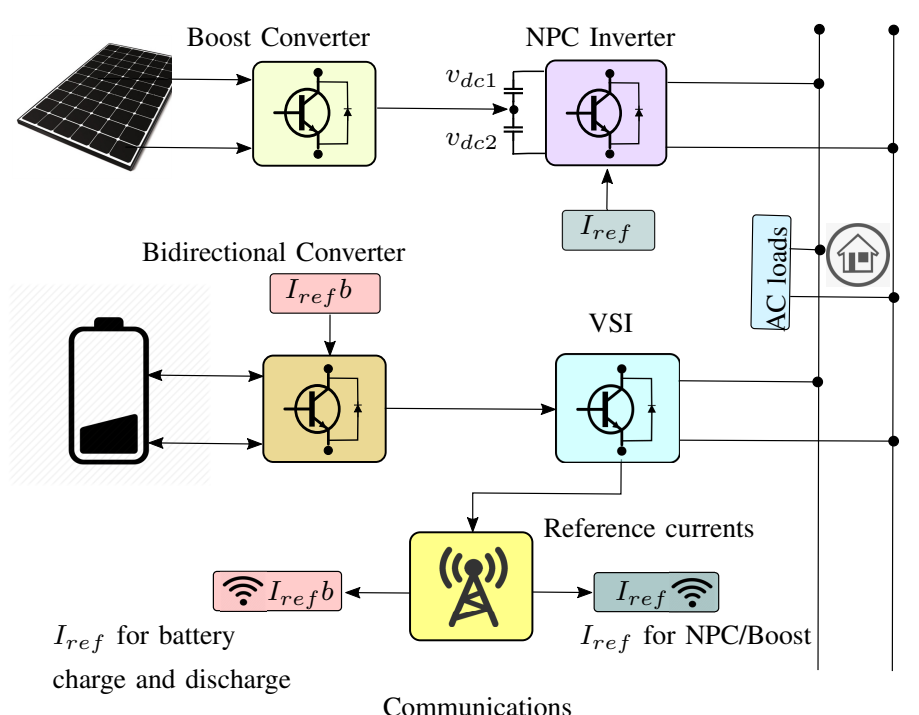

Fig. 1. Diagram of the microgrid cluster. 


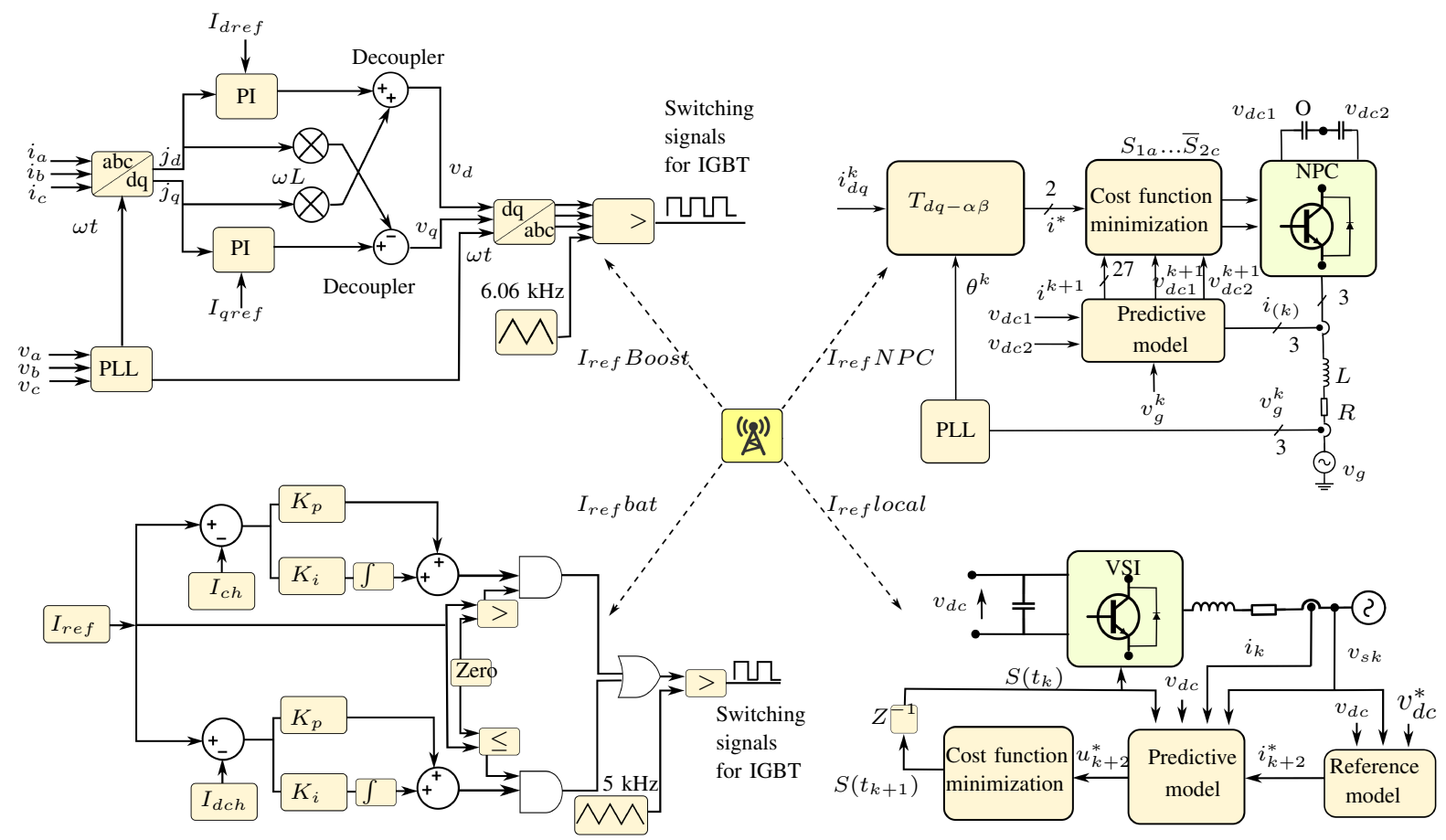

Fig. 2. Control strategy applied to the cluster.

Although this strategy solves particular problems of energy exchange in electric microgrid, it has some drawbacks such as the slow dynamic response and the decrease in optimal coordinated performance. Some of the disadvantages of the droop control founded in the literature [5] are: 1) active and reactive coupling errors due to the variation of the impedance between DG sources; 2) inadequacies in the control strategy when the MG topology is complex; 3) deterioration of the shared current, caused by deviations in voltage and load; 4) presence of circulating currents; 5) does not consider the total cost of generation, or other variables, as the active or reactive power sharing. Thus, the current alternatives have sought more complex microgrids configurations (in loops or meshes), making the exchange of power (especially reactive power), a more challenging task.

The second strategy is based on the control of distributed generation sources from communication devices. Local controllers must be coordinated efficiently, to maintain load balancing and optimize the performance of the power grid. Coordination can be achieved through a centralized, or a distributed architecture. In the centralized control, the data is sent to a MG central controller (MGCC), where it is processed through a predefined control algorithm and then the feedback commands are sent to the local agents through a communication system. On the other hand, in the distributed architecture, each local controller executes certain actions and the final decisions are executed locally in a distributed manner.

Both alternatives (centralized and distributed), allow the design of algorithms for power sharing, achieving optimal performance of the MG, and can function as a complement to another type of control, such as droop control. The main drawback of communication-based control lies in the deterioration of reliability, due to the delay introduced in the system by the communication devices. However, if more sophisticated intelligent algorithms are required, communication control becomes a necessity, due to the lack of real-time information from other strategies.

While several recent research address some operational problems of power exchange with communications [2], [6], it is still necessary to focus efforts on some questions described in [2], [7] include 1) the planning of the MG according to the generation needs, storage and consumption; 2) the control and management of the MG in an autonomous way; 3) adequate coordination and regulation of energy between the MG groups; and 4) the formulation of network policies, which allows the overall operation of the system; 5) the establishment of efficient and reliable control in a microgrid with multiple DGs; 6) communication technology would guarantees better results during the exchange of information and power in the MG; 7) how should noise be modeled? 8) which communication strategy would takes better advantages of the available resources? 9) in the upper layers of the hierarchical model, which intelligent algorithm will favor the optimal power flow between the primary controllers? 10) does the communication system meet the needs of intelligent algorithms? 11) how to integrate all the elements so that the power exchange is improved and does not affect the availability in case of failures?

The need for a reliable communication system, at high transmission speeds through an erratic channel, as in most 
current electricity networks, has been the main motivation of this research. In it, the researchers propose to solve some of the previous questions through a microgrid cluster, with a communication system to share power. Four topologies of power converters are used, each with its local controller and a communications system. The architecture considers two integral proportional classic controllers (PI) and two controllers using model-based predictive control. The properties of the communication channel were calculated according to the characteristics of the communication environment where they are located. The transmission medium used is a wireless communication channel, such as that used in WLAN networks. The analysis of the shared power in each of the architecture buses is outside the scope of this research.

\section{MG CLUSTER ARCHITECTURE}

The architecture of the MG cluster may vary according to multiple elements such as the generation needs, the characteristics of the power converters and the local controllers, or according to the type of bus they share. This research considers an architecture formed by a Boost converter, described in [8] connected to a solar panel. Its output feeds an NeutralPoint Clamped inverter (NPC), which is connected to an AC bus supplying the loads. The control strategy applied to NPC is a predictive control based on models. As in the VSI converter, the control strategy is determined by the cost function. Predictive control strategies were developed by the researchers in this paper. A bidirectional converter connected to a battery is added [8], which, during normal operation, keeps storing energy and during the change to island mode delivers power to the loads. Fig. 2, shows the control strategy applied to the cluster implemented in this investigation. As can be observed, the communications system located in the VSI, sends its references to each controller and from there, each outputs is obtained.

System performance degrades as the intensity of the transmitted symbols is dispersed in the channel. Inter-symbol interference (ISI) appears, which has been treated by several authors [9]. These studies have shown that OFDM (orthogonal frequency division multiplexing), is a viable solution to mitigate the effect of ISI in a computationally efficient way. OFDM is expected to allow high-speed communication, required by intelligent applications. In addition, the high immunity to noise makes this technology an ideal candidate for noisy environments, like the one that exists in electrical networks. There are few references to the use of OFDM for communications in a microgrid cluster, according to different control strategies.

The idea behind OFDM is that, instead of transmitting high-speed data symbols using a carrier, the $N$ blocks of data symbols are transmitted in parallel using $N$ subcarriers, each at a lower speed $(R / N)$. As a result, the length of each subcarrier's symbol can be increased as larger than the channel delay spread $(\tau)$, thus combating the ISI [10].

\section{MODEL OF THE COMMUNICATION CHANNEL}

There are several factors that can attenuate the transmitted signal and degrade its quality in a wireless environment. For example, multipath fading [11] is an effect caused when the signal obtained at the receiver arrives through different paths (product of several reflections), with different intensity and phase. Initially, the impulse response of the system is proposed and subsequently a channel is implemented according to the noise and the estimated conditions.

\section{A. System impulse response}

The impulse response of the system considers the channel as a filter with certain characteristics. In the literature two models are defined to address the effect of multipath due to the complexity of modelling the amplitude of each trajectory accurately. These models are the Rician probability density function and the Rayleigh probability function. The first one describes the route amplitudes when one of them is dominant and not faded (there is at least one line of direct visibility (LOS) between the transmitter and the receiver). For its part, the Rayleigh probability function describes when there are several routes, but none with direct visibility [9]. The filter used to describe the impulse response of the wireless channel is:

$$
\left.h(t, \tau)=\sum_{k=1}^{N(\tau)} \beta_{k}(t) \delta\left[\tau-\tau_{k}(t)\right)\right] e^{j \theta_{k}(t)}
$$

where $t$ is the impulse observation time, $\tau$ is the impulse application time, $N(\tau)$ is the number of multipath components, $\beta_{k}(t)$ is the time randomly varying the amplitude of the trajectory, $\tau_{k}(t)$ is the arrival time and $\theta_{k}(t)$ is the phase. This model will describe the channel completely and the received signal is only the convolution of the $s(t)$ signal with $h(t, t a)$ and the addition of noise.

The distribution of the path amplitude depends on the presence of a LOS path. For this simulation, we will assume that the LOS component is not present and, therefore, we will use the Rayleigh model. This model assumes that there are many smaller independent routes (in magnitude) and that the number of routes is fixed. The delay between routes is equidistant and the phases follow a uniform distribution.

The Rayleigh fade channel model can be calculated as [9]:

$$
P_{R}(r)=\frac{r}{\psi_{0}} e^{\left[-\frac{r^{2}}{2 \psi_{0}}\right]}
$$

where $\psi_{0}$ is the power of the scattered rays and $r$ is the distance. To test the attenuation of the channels provided by the model, the average amplitude (in $\mathrm{dB}$ ) of its frequency response is calculated. The dispersion delay is commonly defined as the mean quadratic value (rms) of the channel power delay (i.e. of the energy distribution of the impulse response). The effective length is defined as the time interval that contains a certain percentage of the impulse response energy, with $90 \%$ being a common value in practice. 


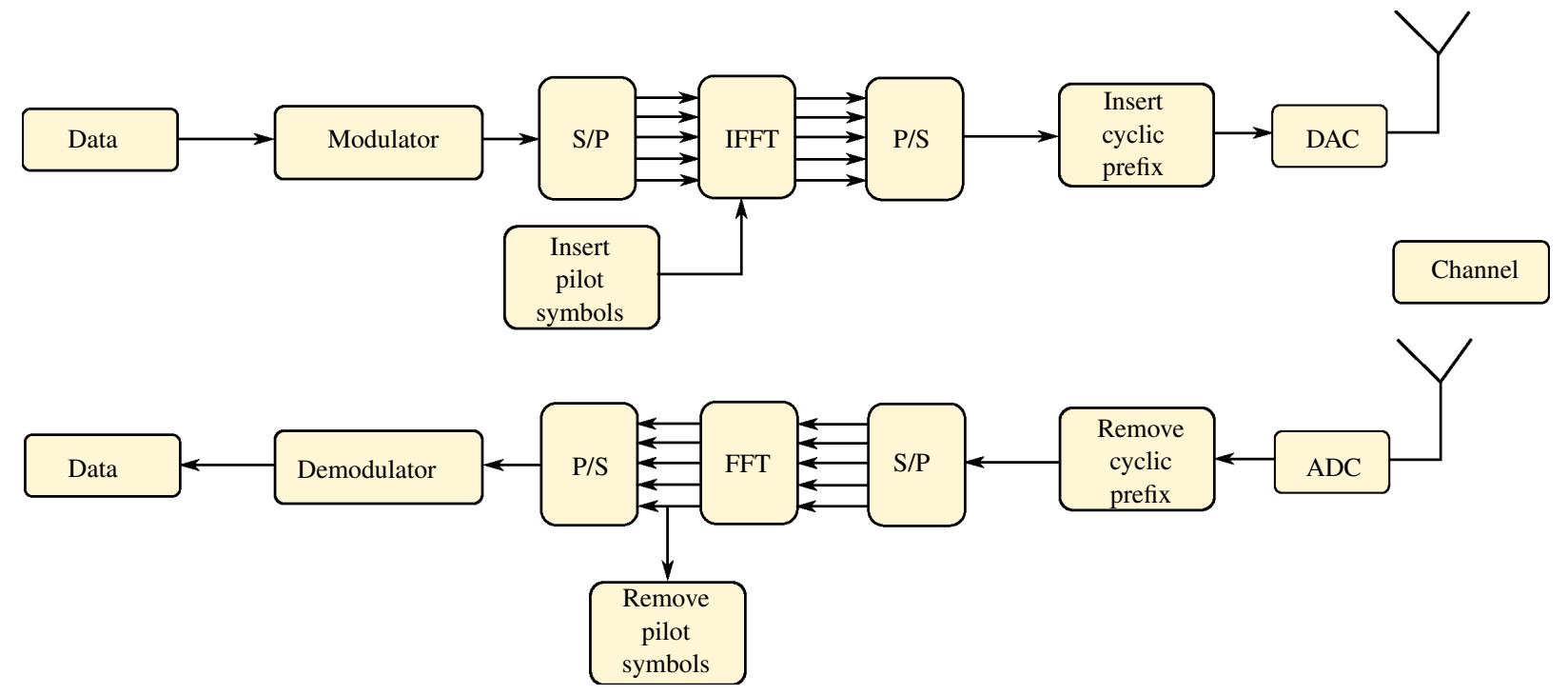

Fig. 3. Communications architecture.

\section{B. AWGN channel estimation}

Our channel model includes, in addition to a Rayleigh probability density model to simulate the multipath effect, and mathematical model of Additive Gaussian White Noise (AWGN). This channel estimation has been frequently used to determine the type of modulation, the order of modulation and the most appropriate comparison between different coding schemes. However, due to its simplicity, AWGN is commonly used to simulate artificial noise or other multi-user interference. The signal received in the time interval $0 \leq t \leq T$ can be expressed by the equation (3):

$$
r(t)=S_{m}(t)+n(t)
$$

where $r(t)$ is the received signal, $S_{m}$ is the transmitted signal and $n(t)$ is the sample function of the AWGN process with power spectral density. The cumulative distribution function (CDF) of a random variable with Gaussian or distributed distribution can usually be presented as the equation (4) [11]:

$$
F(x)=\frac{1}{2}\left[1+\operatorname{erf}\left(\frac{x-\mu}{\sqrt{2 \sigma^{2}}}\right)\right]
$$

In order to get closer to the reality, a noisy channel as in most electrical environments, is defined.

\section{Phase noise model}

Phase noise and time fluctuation in oscillators and Phase Locked Loop (PLL) are a major concern in wireless and optical communications, since they contribute significantly to the bit error rate of communication systems and create synchronization problems in digital systems of sampled and synchronized data. Generally during the last decade, phase noise and time fluctuation received intense attention from researchers in various fields, from analog / RF circuit design and computer-aided design (CAD), to optical / wireless communications and applied mathematics. A good theoretical and practical understanding of noise in oscillators and PLLs has been achieved.

\section{Proposed Communications Architecture}

The communications architecture used in this proposal considers two modulation techniques for OFDM and compares the performance of each of them according to the bit error rate (BER) and the energy per bit / spectral density ratio of noise power $\left(E_{b} / N_{0}\right)$. The proposal is summarized in Fig. 3, where the data bit streams (signal sent from the controller) are converted from series to parallel and each subcarrier is modulated using QPSK and 64 QAM. Next, they are converted from series to parallel to be transported by the OFDM carrier and then pass through the IFFT module, to create a complex signal that contains all the subcarriers. The parallel data stream is converted into a serial stream and the real and

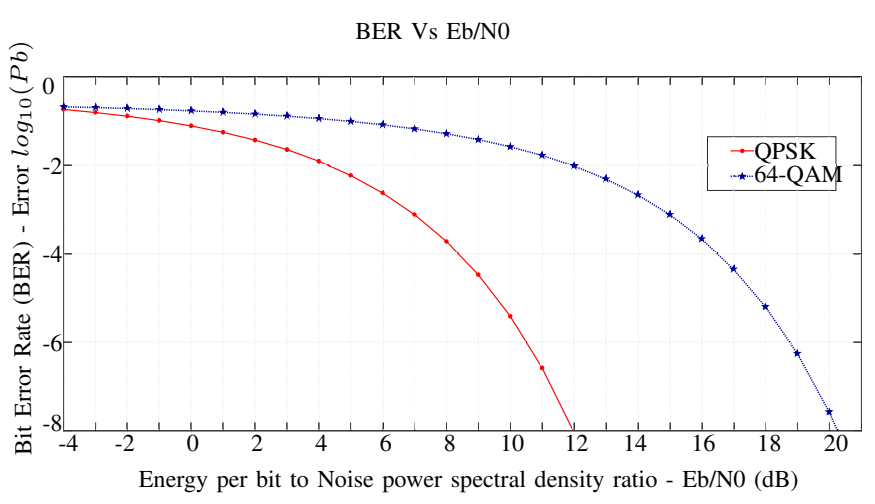

Fig. 4. Performance analysis of QPSK and 64 QAM modulation techniques. 
imaginary signal is processed in a digital analog converter to be transmitted by the antenna.

The received signal is obtained many times due to the propagation of multiple paths, especially in an open and irregular environment like the simulated one. The line of sight and the multipath signal have a different arrival time. The OFDM scheme proposes to reduce the ISI effect as long as it can preserve orthogonality. Orthogonality can be achieved with the addition of guard time (guard interval). The save time in OFDM was achieved by inserting zero padding (ZP), or a cyclic prefix (CP). The cyclic prefix extends the OFDM symbol, copying the last samples of the OFDM symbol, on its front. In this way, the $\mathrm{CP}$ is entered before the OFDM symbol.

\section{RESULTS AND DISCUSSION}

The simulation model is designed for the configuration of a dispersive channel with a Rayleigh probability distribution, added to an AWGN channel with phase noise. Two widely used OFDM modulation techniques are therefore employed, and the results are compared. A VSI converter is proposed within the microgrid cluster, which is controlled by the predictive control algorithm. Ideally, to control the microgrid, an MGCC is required that is capable of controlling all the devices on the network. To simplify the study, it was defined that the predictive control of the VSI who determines the reference values that will be sent to the DC-DC bidirectional converter, the NPC and the Boost converter. The VSI will be connected to the OFDM transmitter, which will simulate the communication process. According to this assumption, a receiver must be installed in each converter to receive the communication signal.

The performance of the system using the communication strategy for energy exchange is shown in Fig. 5. The performance of the cluster is not affected by the communication strategy, despite being in a very noisy communications channel. In addition, the reference is modified by the central microgrid controller, but each converter can adjust its output, through its control strategy. This adds greater redundancy to the microgrid, and allows balancing the loads and guaranteeing the exchange of power.

The value sent by the MGCC is the three-phase reference current amplitude value for the NPC converter. According to the simulated environment, this value changes every $20 \mathrm{~ms}$, simulating that during that time, the central controller indicates that the output current must adjust its value, to favor the exchange of power. Fig. 5 (c) shows the output current (above) and the voltage in one of the phases of the NPC converter. Thus, there is a change in the reference to 20 milliseconds, whose value is received through the communications system. Furthermore, the MGCC also sends the reference voltage for the DC link capacitors and whose values are shown in Fig. 5 (d).

When the exchanged power reaches the desired value, the communication system can stop sending information and in each of the local controllers it will use the last reference sent by the communication system. If it is necessary to increase the power, it will be crucial to restart the communication system to adjust the reference values. Once the new reference is received, the local controller will adjust the output of the converter, without exceeding the allowed values set in the control strategy.

On the other hand, the central controller sends the reference current for the bidirectional converter. In this case, a change of operating mode is simulated at 40 milliseconds, moving from storing energy to delivering power to the network. This behavior can be seen in Fig. 5 (a). Finally, the reference of the Boost converter connected to the solar panel is also sent from the MGCC located as part of the VSI converter predictive control model. Every 20 milliseconds, the converter is instructed to adjust the output current, simulating a fault in one of the converters, and causing an increase in the output current of the Boost converter. This behavior is seen in Fig. 5 (b).

The hardware limitations depend on the communication devices used in the system. The inclusion of a communication system within a control strategy degrades the reliability of the entire proposed architecture. However, some of the most recent advances in control strategies, such as multi-objective control strategies [12], require real-time data, which can only be achieved with communication devices.

The probability of bit transmission error is plotted against the ratio between the energy per bit of the transmitted signal and the spectral density of noise power $\left(E_{b} / N_{0}\right)$. According to the transmitted sequence, clearly 64 QAM has better performance, as shown in Fig. 4. This behavior is justified because it has a higher signal-to-noise ratio $\left(E_{b} / N_{0}\right)$ for the established bandwidth. It is relevant to notice that in the simulations performed with QPSK, an error of $0.61 \%$ of lost packets is obtained, for a total of $2.19 * 10^{6}$ bits transmitted. This result is not entirely poor, however, the high influence of intersymbol interference in the modulation strategy indicates that in environments with multiple reflections, it is not the ideal alternative.

On the other hand, for the same amount of bits, an error of $0.46 \%$ is reached, for OFDM with 64 QAM modulation. Hence, the BER (bit error rate) remains at well below 1, which indicates the effectiveness of the technology and its robustness against interference in very noisy channels such as electrical channels. In this sense, this modulation strategy improves the results of its QPSK competitor, making it a tempting alternative for this type of applications. The results obtained can be replicated in other case studies, where the inter-symbol interference is very high and the typical channel noise affects the communication, as the most power systems.

\section{CONCLUSIONS}

In this investigation, an OFDM communications strategy was implemented, based on two modulation techniques. It has been possible to verify that the predictive control is capable of managing the exchange of power in a microgrid cluster and sending the reference values from the communications system. 


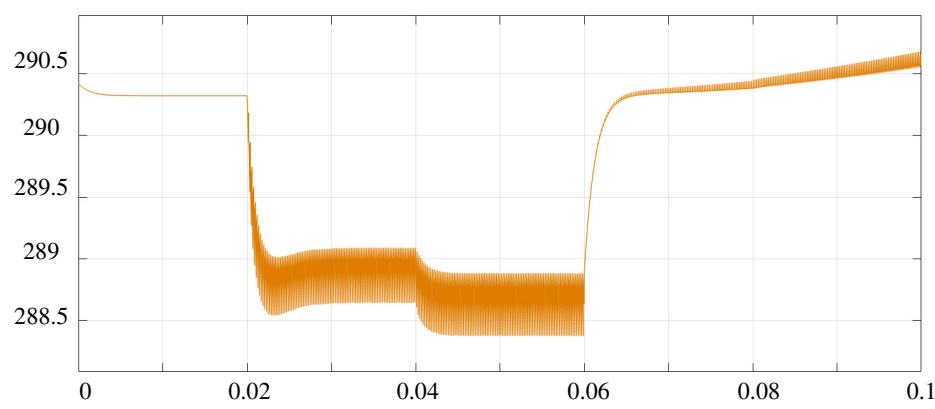

(a) Voltage on the DC bus of the bidirectional converter

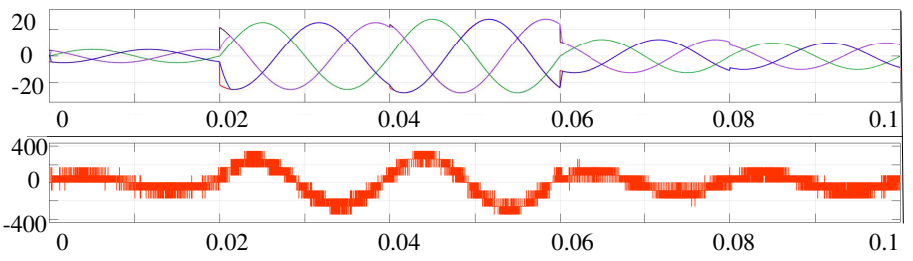

(c) Output voltage of the NPC converter and switching states in one phase.
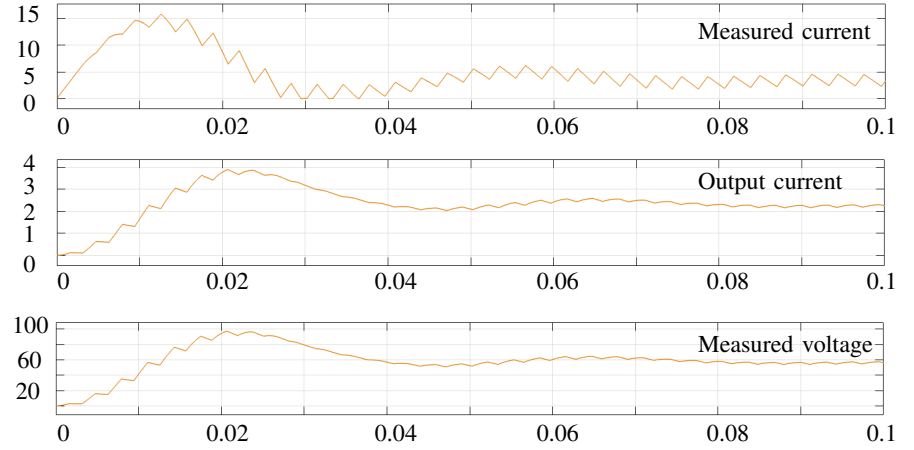

(b) Boost converter output current and voltage

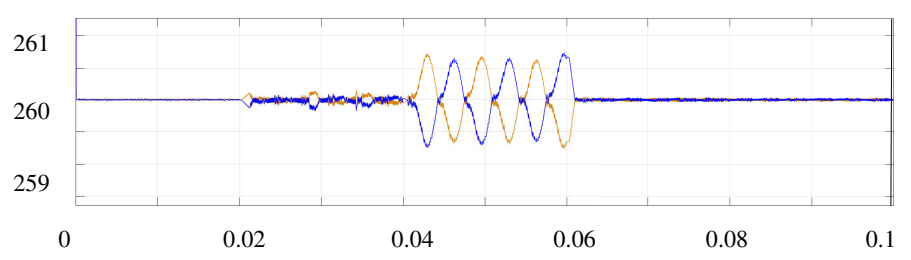

(d) Voltage balance of DC link capacitors in NPC converter

Fig. 5. Output of each converter using the proposed communications system.

In addition, due to the use of a communication strategy, restrictions for the exchange of energy are eliminated when different control strategies were used, such as the case of fall control. It was found out that the predictive control can exchange energy efficiently with another controller using the same strategy, or with a classic PI control. OFDM communication technology is a very strong candidate to establish communication between power converters, due to the high immunity against noise and the reduction of inter-symbol interference. The transmission error was very low, despite using a noisy channel model. Finally, the impact of grouping multiple microgrids has been investigated and it has been proven that the joint operation of them allows to increase the efficiency of the network in terms of bit error rate and signal to noise ratio. As a future work, we expect to develop a communication prototype based on OFDM for power sharing in a laboratory microgrid cluster. This implementation will allow the development of new intelligent control strategies in the upper layers of the hierarchical control model.

\section{ACKNOWLEDGMENTS}

The authors wish to thank the FONDECYT Regular Program through project 1160690 and the CONICYT National Doctorate Scholarship 2019, folio 21190709.

[2] L. Meng, Q. Shafiee, G. F. Trecate, H. Karimi, D. Fulwani, X. Lu, and J. M. Guerrero, "Review on control of dc microgrids and multiple

\section{REFERENCES}

[1] T. Dragičević, X. Lu, J. C. Vasquez, and J. M. Guerrero, "Dc microgrids-part ii: A review of power architectures, applications, and standardization issues," IEEE transactions on power electronics, vol. 31, no. 5 , pp. $3528-3549,2015$. microgrid clusters," IEEE Journal of Emerging and Selected Topics in Power Electronics, vol. 5, no. 3, pp. 928-948, 2017.

[3] E. Bullich-Massagué, F. Díaz-González, M. Aragüés-Peñalba, F. GirbauLlistuella, P. Olivella-Rosell, and A. Sumper, "Microgrid clustering architectures," Applied Energy, vol. 212, pp. 340-361, 2018.

[4] Y. Han, H. Li, P. Shen, E. A. A. Coelho, and J. M. Guerrero, "Review of active and reactive power sharing strategies in hierarchical controlled microgrids," IEEE Transactions on Power Electronics, vol. 32, no. 3, pp. 2427-2451, 2016.

[5] J. M. Guerrero, J. C. Vasquez, J. Matas, L. G. De Vicuña, and M. Castilla, "Hierarchical control of droop-controlled ac and de microgrids-a general approach toward standardization," IEEE Transactions on industrial electronics, vol. 58, no. 1, pp. 158-172, 2011.

[6] R. E. Pérez-Guzmán, Y. Salgueiro-Sicilia, and M. Rivera, "Communication systems and security issues in smart microgrids," in Power Electronics Conference (SPEC), 2017 IEEE Southern. IEEE, 2017, pp. 1-6.

[7] T. Dragičević, X. Lu, J. C. Vasquez, and J. M. Guerrero, "Dc microgrids-part ii: A review of power architectures, applications, and standardization issues," IEEE transactions on power electronics, vol. 31, no. 5, pp. 3528-3549, 2016.

[8] M. Saleh, Y. Esa, Y. Mhandi, W. Brandauer, and A. Mohamed, "Design and implementation of ccny dc microgrid testbed," in 2016 IEEE Industry Applications Society Annual Meeting. IEEE, 2016, pp. 1-7.

[9] I. Jarin and R. Sharmin, "Performance evaluation of siso ofdm system in the presence of cfo, timing jitter and phase noise for rayleigh and rician fading channels," in 2017 IEEE Region 10 Humanitarian Technology Conference (R10-HTC). IEEE, 2017, pp. 498-501.

[10] C. Kotchasarn, "Performance analysis of broadband power line communications with ofdm transmission," in Advanced Communication Technology (ICACT), 2017 19th International Conference on. IEEE, 2017, pp. 316-320.

[11] H. Schulze and C. Lüders, Theory and applications of OFDM and CDMA: Wideband wireless communications. John Wiley \& Sons, 2005.

[12] L. Meng, E. R. Sanseverino, A. Luna, T. Dragicevic, J. C. Vasquez, and J. M. Guerrero, "Microgrid supervisory controllers and energy management systems: A literature review," Renewable and Sustainable Energy Reviews, vol. 60, pp. 1263-1273, 2016. 